\title{
Primary pulmonary arterial sarcoma treated with Endostar injection and radiotherapy
}

\author{
Hui Xu, MD, Wenyu Yang, MD, Chengyun Wu, MD.
}

\begin{abstract}

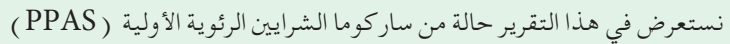

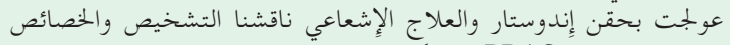

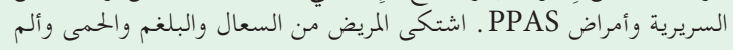

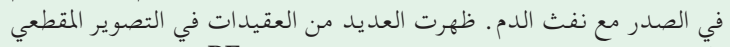

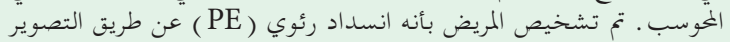

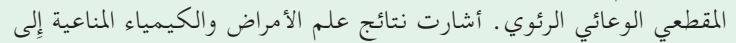

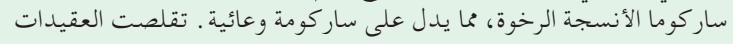

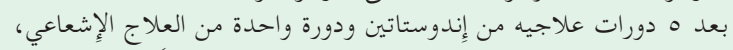

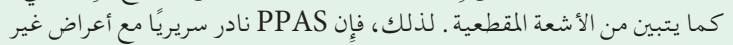

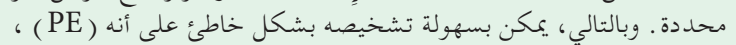

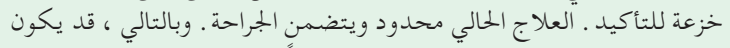

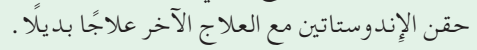

A case of primary pulmonary arterial sarcoma (PPAS) treated with endostar injection and radiotherapy and discuss the diagnosis, clinical characteristics and pathology of PPAS. The patient complained of cough, sputum, fever, and chest pain with hemoptysis. Numerous nodules were seen in the computed tomography scan. The patient was diagnosed as pulmonary embolism (PE) by computed tomography pulmonary angiography. The pathology and immunohistochemistry results indicated soft tissue sarcomas, indicative of angiosarcoma. The nodules shrunk after 5 courses of endostatin and one course of radiotherapy, as seen by CT scan. Therefore, PPAS is clinically rare with nonspecific symptoms. Hence, it can be easily misdiagnosed as PE, biopsy for confirmation. Current treatment is limited and includes surgery. Hence, endostatin injection combined with other therapy may be an alternative treatment.

Keywords: PPAS, endostatin, radiotherapy

Saudi Med J 2020; Vol. 41 (4): 421-425 doi: $10.15537 /$ smj.2020.4.25010

From the Department of Respiratory Medicine, the Second Affiliated Hospital of Wenzhou Medical University, Wenzhou, China.

Received 16th June 2019. Accepted 4th December 2019.

\begin{abstract}
Address correspondence and reprint request to: Dr. Chengyun Wu, Department of Respiratory Medicine, the Second Affliated Hospital of Wenzhou Medical University, Wenzhou, China. E-mail:wcy857516126@163.com

ORCID ID: http://www.orchid.org/0000-0001-5142-8275
\end{abstract}

$\mathrm{P}$ rimary pulmonary arterial sarcoma (PPAS) is a rare malignant soft tissue tumor. To date, less than 300 cases have been reported. Due to the lack of specific symptoms and physical signs, PPAS is difficult to diagnose, and is easily misdiagnosed as pulmonary thromboembolism (PTE). The majority of cases need surgery or autopsy for confirmation. Comprehensive treatment including surgery is considered, but the curative effect remains poor and unsatisfactory due to a very few reported cases.

Herein, we present a case of PPAS treated with endostar injection adjuvant to radiotherapy, resulting a good curative effect (Figure 1). This case will facilitate proper treatment of PPAS in the future.

Case Report. We present the case of a 34-year-old male patient who was diagnosed with PPAS. The patient's written informed consent was obtained before admission. The patient was admitted to our hospital due to cough and hemoptysis with chest pain for one month. The patient developed a cough without any obvious cause, with yellow-green purulent sputum, blood in phlegm, intolerable persistent pain in the right side of the chest during inhalation and coughing, with chills and fever, shortness of breath after activity relieved after rest.

Clinical findings. Physical examination diagnosed that breath sound was coarse with no rales. Heart rhythm was normal, and no edema. Chest computed tomography (CT) (Figure 2) showed multiple nodules on the cupula pleura of the right lung. Cardiac ultrasound showed moderate pulmonary hypertension with mild mitral valve regurgitation, and mild pulmonary valve regurgitation. Computed tomography 
Male, 34 years old, was previously healthy.

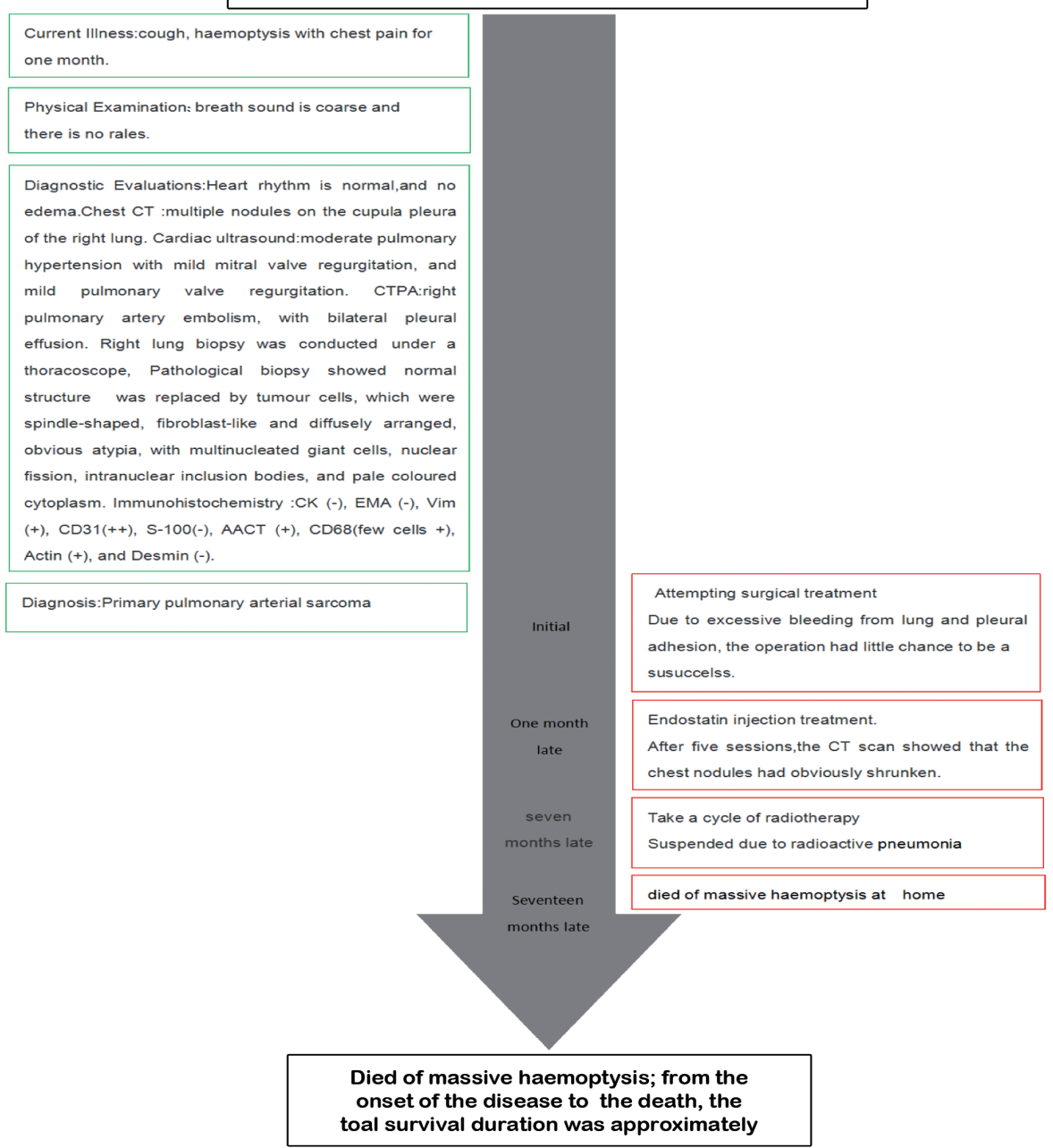

Figure 1 - Timeline flowchart of the patient with primary pulmonary arterial sarcoma. CTPA: computed tomography pulmonary angiogram

pulmonary angiography showed right pulmonary artery embolism, with bilateral pleural effusion. The deep vein ultrasound of lower limbs showed no abnormalities. Two percutaneous lung biopsies were negative. Right lung biopsy was conducted under a thoracoscope at our hospital. Postoperative pathological biopsy (Figure 3) showed normal structure replaced by tumor cells, which were spindle-shaped, fibroblastlike and diffusely arranged. In addition, it showed obvious atypia with multinucleated giant cells, nuclear fission, intranuclear inclusion bodies, and pale colored

Disclosure. Authors have no conflict of interests, and the work was not supported or funded by any drug company. cytoplasm. Immunohistochemistry showed CK', EMA', $\mathrm{Vim}^{+}, \mathrm{CD}^{3} 1^{++}, \mathrm{S}-100^{-}$, AACT ${ }^{+}$, a few cells were CD68 ${ }^{+}$, actin $^{+}$, and desmin'. In fact, AACT and CD68 do not refer to tumor cells, but to the surrounding stromal cells. Because the whole lesion is cut off when the operation is performed, there are tumors and surrounding tissues in the pathological section.

Diagnostic assessment. Pathology and immunohistochemistry results indicated characteristic soft tissue sarcomas, indicative of angiosarcoma according to clinical symptoms, CT imaging features and pathological biopsy results. We have examine the whole body but have not found other site of angiosarcoma. The patient was diagnosed as PPAS.

Therapeutic intervention. The patient was recommended for surgery as the treatment of choice. 


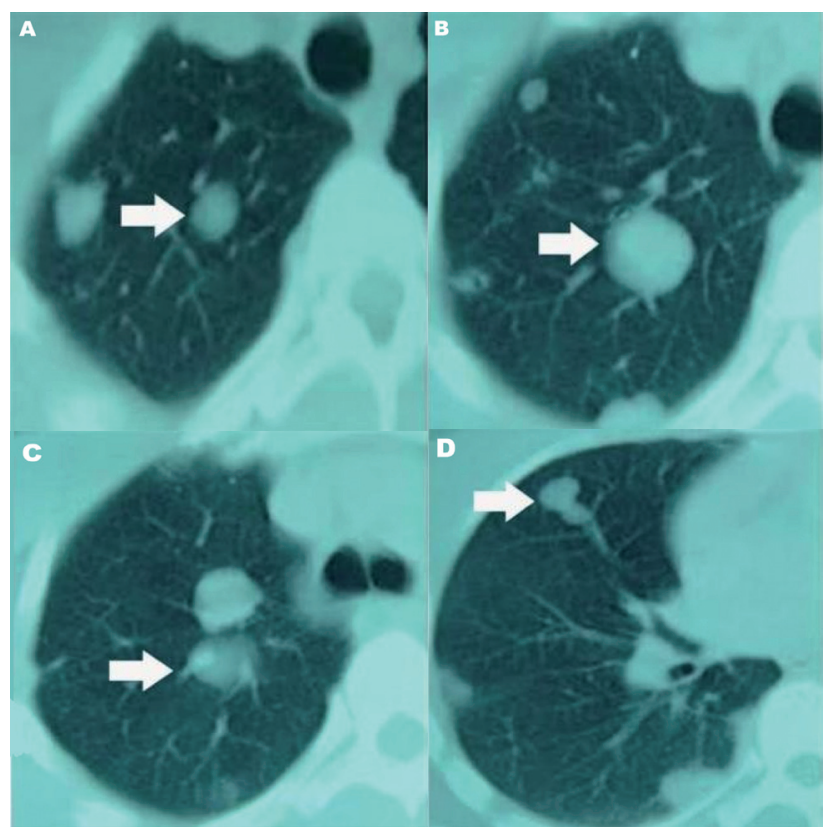

Figure 2 - Multiple nodules of unequal size in the right lung, with involvement of pleura. A,C) Nodules in upper lobe of right lung, B) nodules in upper lobe of right lung with involvement of pleura, and D) nodule in middle lobe of right lung.
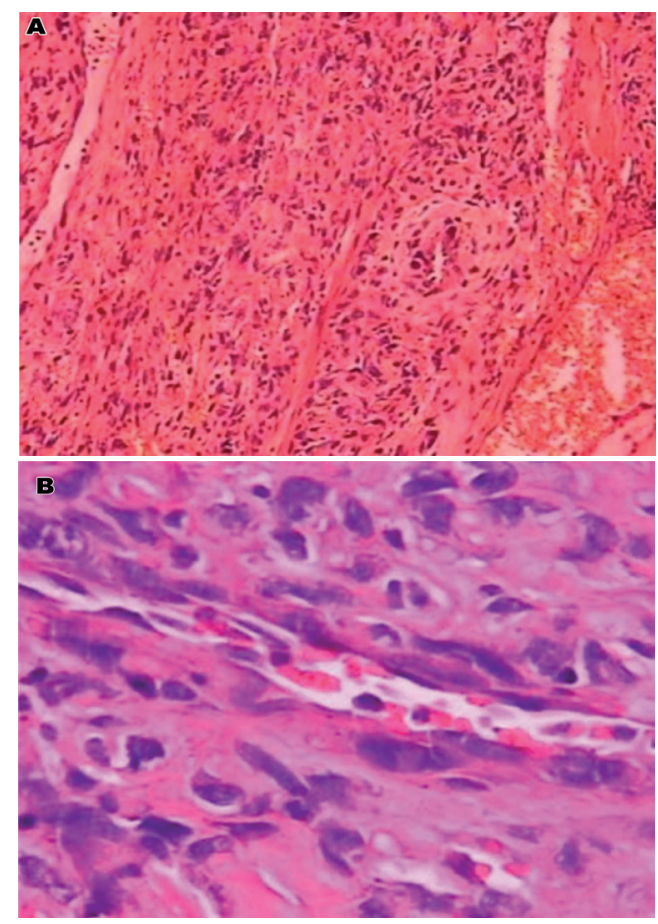

Figure 3 - Tumor was composed of fibroblast-like spindle cells, with obvious atypia and pale cytoplasmic eematoxylin and eosin staining. A) Tumor was composed of atypia fiber-like spindle cells,HEx100 and B) the tumor cells were obviously atypia, and the nuclei are irregular were atypia, HE×400.
Due to excessive bleeding from lung and pleural adhesion, the operation could not be a success. The patient declined chemotherapy and received 15 $\mathrm{mg} /$ day endostatin injection for 14 days. Computed tomography scan was repeated after 5 sessions (Figure 4), which showed that the chest nodules had obviously shrunken. A cycle of radiotherapy was initiated after 6 months. Radiotherapy was carried out only once at 200 cGy/F, external location 2 to $3 \mathrm{~cm}$ from the local lesion field. Due to radioactive pneumonia, the patient only received radiotherapy once. Chest CT was repeated (Figure 5), which showed the nodules had significantly shrunken. Nine months after radiotherapy, another chest CT indicated that the lesions had progressed and spread to the left shoulder joint.

Follow-up and outcomes. The patient died of massive hemoptysis at his home after about a month. From the onset of the disease to the death, the total survival duration was approximately 18 months.

Discussion. Angiosarcoma is a malignant tumor originating from the vascular endothelium. Primary pulmonary arterial sarcoma is one of angiosarcomas that grow within the pulmonary artery. The World Health Organization (WHO) classified PPAS into wall sarcomas (mainly leiomyosarcoma) and endometrial sarcomas, which are most prevalent. ${ }^{1}$ Symptoms usually include progressive dyspnea, cough or hemoptysis, and chest pain, sometimes accompanied by fever. Blocked blood flow caused by mass growth within the pulmonary artery, result in similar pathophysiological changes and clinical manifestations as PE, which makes the differential diagnosis difficult. ${ }^{2}$ Computed tomography pulmonary angiography indicating pulmonary artery wall erosion is a characteristic feature. ${ }^{3}$ The key differences between PE and PPAS are that: PPAS most commonly occurs in the main pulmonary artery or proximal pulmonary artery, with often visible complete filling defect, its clinical symptoms do not involve anticoagulation mechanism; no thrombus source; multiple nodules in lung parenchyma; lumen outside the cavity is affected; and visible swollen lymph nodes in the mediastinum and hilum; PPAS is often a unilateral disease, while PE shows bilateral lesions; also, PPAS can show uneven enhancement due to tumor angiogenesis. ${ }^{4}$ Primary pulmonary arterial sarcomahas high degree of malignancy and poor prognosis, with an average survival time of 13 to 18 months. A 1-year survival rate of $22 \%$ and 2 -year survival rate for $7 \% .^{5}$ Without surgical intervention, the average survival time is only 1.5 months after diagnosis. ${ }^{6}$ 

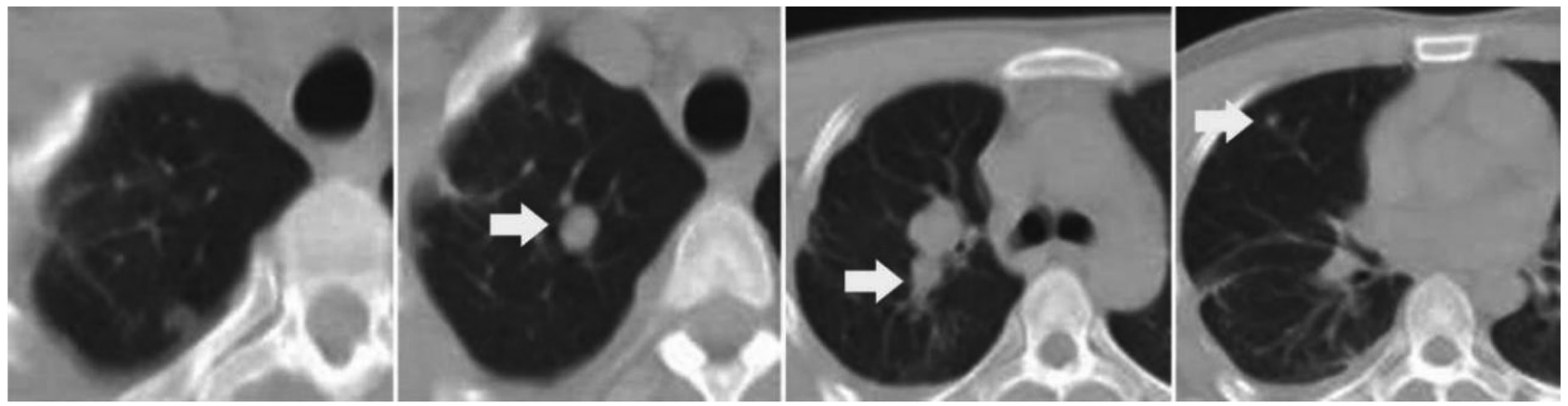

Figure 4 - Pulmonary nodules significantly shrink after treatment with endostar.
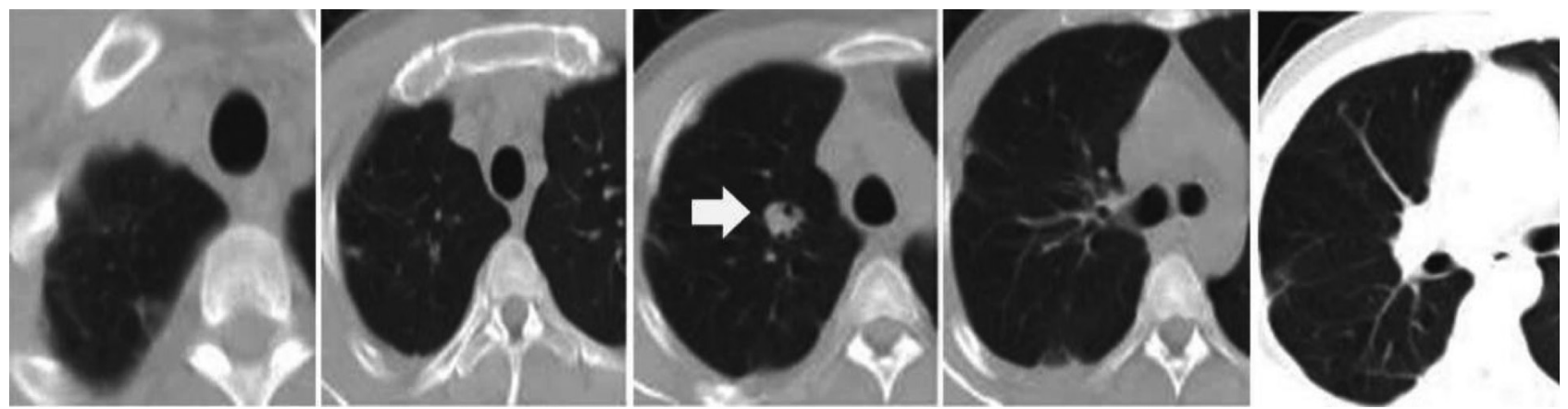

Figure $\mathbf{5}$ - Nodules significantly shrink after radiotherapy.

There is no ideal treatment strategy for PPAS. The preferred treatment is complete surgical resection. Pneumonectomy is only suitable for unilateral pulmonary artery involvement. If sarcoma infiltrates outside the walls of blood vessels, pneumonectomy should be performed for unilateral lesion but not for bilateral lesions. Chemotherapy and radiotherapy are considered for patients with contraindication to surgery. But their efficacy remains unclear due to the absence of large sample clinical studies. There are no standard chemotherapy regimens for pulmonary artery sarcoma.

Penel et $\mathrm{al}^{7}$ reported 7 pulmonary artery sarcoma patients with failed doxorubicin chemotherapy. Hirose et $\mathrm{al}^{8}$ reported recurrence in a postoperative patient treated with amrubicin hydrochloride and carboplatin with a poor curative effect but a good radiation effect.

Dacarbazine, ifosfamide, mesna and etoposide scheme may have certain curative effects on PPAS. ${ }^{9}$ Other treatment options include adriamycin, ifosfamide, carboplatin, and paclitaxel.

Endostar, a human recombinant endostatin injection, can inhibit the tumor growth by inhibiting tumor angiogenesis, with less toxic side-effects. Its first-choice indications are chemotherapy regimens (plus vinorelbine and cisplatin, NP) for the initial treatment or retreatment of stage III/IV non-small cell lung cancer patients. By analysis 9 randomized controlled trials and cohort studies, Zhou et a ${ }^{10}$ found that endostatin combined chemotherapeutic agents significantly improved clinical efficiency compared with chemotherapeutic agents alone in treating bone and soft tissue sarcomas(include gstrointestinal stromal tumors, unclassified sarcomas, liposarcomas, leiomyosarcoma, synovial sarcoma and malignant peripheral nerve sheath tumor,etc ).

In this case study, we reported for that endostatin was effective in treatment of PPAS. Endostar was chosen for study because, having broad-spectrum anti-angiogenic activity and inhibits tumor neovascularization by inhibiting the migration of endothelial cells forming blood vessels. For PPAS, proliferation or metastasis of malignant vascular endothelial cells may be inhibited by endostar treatment. The patient received only one dose of radiotherapy, which is not effective for radiotherapy except for treatment of the follow-up effects of angiogenesis inhibitors.

Endostar is not currently been used for the treatment of PPAS. We have shown that it had curative effects in our patient, although the underlying mechanism remains unknown. It has a broad-spectrum 
anti-angiogenesis activity by inhibiting vascular endothelial cell migration, which then inhibits tumor proliferation. Pulmonary artery sarcoma exhibits high levels of estimated glomerular filtration rate, platelet derived growth factor receptor alpha, and mouse double minute 2; whether endostar plays a role for these targets remains unclear and needs further study. Primary pulmonary arterial sarcoma is also sensitive to radiation therapy.

Primary pulmonary arterial sarcoma is clinically rare, has nonspecific symptoms, and is easily misdiagnosed as PE. It often requires a biopsy for confirmatory diagnosis. Treatment options are limited.

In conclusion, in addition to surgery, endostar combined with chemotherapy or radiotherapy may be an alternative option.

Acknowledgment. We would like to thank Suchita Bhattacharyya for English language editing; JianMin Chen for clarity and correctness of the figures; and Lan Wang for pathology diagnosis.

\section{References}

1. Andrushchuk U, Mofeejuddy I, Spirydonau S, Yudina O, Shchatsinka M. Surgical treatment of primary pulmonary sarcoma disseminated to both pulmonary arteries using composite pulmonary trunk and bifurcation of abdominal aorta allograft. Kardiochir Torakochirurgia Pol 2018; 15: 254-257.

2. Deng L, Zhu J, Xu J, Guo S, Liu S, Song Y. Clinical presentation and surgical treatment of primary pulmonary artery sarcoma. Interact Cardiovasc Thorac Surg 2018; 26: 243-247.
3. Shingaki M, Kobayashi Y. Bilateral pulmonary artery occlusion due to primary pulmonary artery sarcoma; report of a case. Kyobu Geka 2014; 67: 575-577.

4. Lee Y, Kim HJ, Yoon H, Choi CM, Oh YM, Lee SD, et al. Clinical characteristics and treatment outcomes of primary pulmonary artery sarcoma in Korea. J Korean Med Sci 2016; 31: 1755-1760.

5. Desmarais P, Laskine M, Caporuscio C. Primary pulmonary artery angiosarcoma mimicking pulmonary embolism in a 66-year-old man with dyspnea. CMAJ 2016; 188: E509-E512.

6. Siordia JA, Garlish A, Truong H. Status post pneumonectomy for pulmonary artery sarcoma. BMJ Case Rep 2015; 2015. pii: bcr2015210622.

7. Penel N, Taieb S, Ceugnart L, Dansin E, Hoguet D, Vanseymortier L, Lartigau E. Report of eight recent cases of locally advanced primary pulmonry artery sarcoma: failure of Doxorubicin-based chemotherapy. J Thorac Oncol 2008; 8: 907-911.

8. Hirose T, Ishikawa N, Hamada K, Inagaki T, Kusumoto S, Shirai $\mathrm{T}$, et al. A case of intimal sarcoma of the pulmonary artery treated with chemoradiotherapy. Intern Med 2009; 4 : 245-249.

9. Nowacka A, Muller O, Hurni M, Prêtre R, Niclauss L. Obstructive pulmonary artery sarcoma. Heart Lung Circ 2015; 24: e63cf-e64cf.

10. Ma Z, Guo L, Cui X, Liu H, Liu L. Rh-endostatin concomitant with chemotherapy versus single agent chemotherapy for treating soft tissue and bone sarcomas: a systematic review and meta-analysis. J Pharm Pharm Sci 2018; 21: 386-397.

\section{Ethical Consent}

All manuscripts reporting the results of experimental investigations involving human subjects should include a statement confirming that informed consent was obtained from each subject or subject's guardian, after receiving approval of the experimental protocol by a local human ethics committee, or institutional review board. When reporting experiments on animals, authors should indicate whether the institutional and national guide for the care and use of laboratory animals was followed. 